\title{
The isomorphism problem for residually torsion-free nilpotent groups
}

\author{
Gilbert Baumslag and Charles F. Miller III
}

\begin{abstract}
Both the conjugacy and isomorphism problems for finitely generated nilpotent groups are recursively solvable. In some recent work, the first author, with a tiny modification of work in the second author's thesis, proved that the conjugacy problem for finitely presented, residually torsion-free nilpotent groups is recursively unsolvable. Here we complete the algorithmic picture by proving that the isomorphism problem for such groups is also recursively unsolvable.
\end{abstract}

Mathematics Subject Classification (2000). Primary 20F10; Secondary 20F05, 20F19, 20E06.

Keywords. Finitely presented group, isomorphism problem, residually nilpotent.

\section{Introduction}

1.1. The main theorem. The objective of this paper is to prove the following theorem.

Theorem A. There exists a recursive class of presentations of finitely presented residually torsion-free nilpotent groups with unsolvable isomorphism problem.

More precisely we will prove the

Theorem $\mathbf{A}^{\prime}$. Let $\Pi$ be the collection of all presentations of extensions of the free group $F=\left\langle s_{1}, \ldots, s_{n}\right\rangle$ by the free group $T=\left\langle t_{i}, \ldots, t_{m}\right\rangle$ with $n \cdot m$ defining relations of the form

$$
t_{i}^{-1} s_{j} t_{i}=s_{j} c_{i j} \quad \text { where } c_{i j} \in[F, F]
$$

for $i=1, \ldots, m$ and $j=1, \ldots, n$. Then the isomorphism problem for the groups presented in $\Pi$ is recursively unsolvable. Each group presented in $\Pi$ has solvable word problem and is residually torsion-free nilpotent. 
It is worth noting that the class $\Pi$ is indeed recursive. One has only to single out, as J. Nielsen did (see, e.g., [8]), those presentations in which the images $s_{j} c_{i j}$ of the given generators generate the free group $F$. Moreover, the residual torsionfree nilpotence of the groups defined by $\Pi$ follows from the fact that free groups are residually torsion-free nilpotent [9] once one observes that $T$ acts like the identity on the abelianization of $F$ (for details, see Lemma 2, p. 302 of [1]).

One of the by-products of the approach that we have taken here is the following theorem, which does not seem to immediately follow from the usual Adian-Rabin construction.

Theorem B. If $n$ is chosen sufficiently large, then there is no algorithm to determine of a finite presentation with $n$ generating symbols whether or not the group presented is free abelian of rank $n$.

So it follows that if we consider all finite presentations then there is no algorithm to decide which of the groups so presented are free abelian on the given generators.

1.2. One of the consequences of Theorem $A$. The isomorphism problem for finitely generated nilpotent groups is solvable [5], [6]. It follows then from Theorem A that there exist infinitely many, finitely presented, residually torsion-free nilpotent groups, no two of which are isomorphic, with the same nilpotent factor groups. The special case of the existence of finitely presented, residually nilpotent groups with the same nilpotent images as a free group, the so-called parafree groups, exist in profusion. They have been the subject of various papers (see, e.g., [2] and the references there cited).

1.3. Some general comments. Finitely generated residually nilpotent groups can be viewed as generalizations of finitely generated nilpotent groups and as such it makes sense to compare them with these finitely generated nilpotent groups. Finitely generated nilpotent groups are finitely presented and have solvable word, conjugacy and isomorphism problem. However there are continuously many finitely generated residually torsion-free nilpotent groups and so most of them are not finitely presented [1]. It follows from the fact that finitely generated nilpotent groups have solvable word problem that finitely presented residually nilpotent groups also do. However, as noted in the abstract, there exist finitely presented residually nilpotent groups with unsolvable conjugacy problem. The remaining open algorithmic problem is now settled by Theorem A.

1.4. A sketch of the proof. The basic idea involved in both the construction of a finitely presented residually nilpotent group with unsolvable conjugacy problem and a recursive family of such finitely presented residually nilpotent groups with 
unsolvable isomorphism problem is contained in the thesis of the second author [12]. Miller invented a method whereby a given finite presentation

$$
P=\left\langle s_{1}, \ldots, s_{n} \mid r_{1}, \ldots, r_{m}\right\rangle
$$

of a group $H$ is transformed, by what we term here a word-conjugacy transformation $\omega$, into a presentation $\omega(P)$ of a group $\omega(H)$. The complexity of the word problem, as well as the conjugacy problem, in the group $H$ defined by $P$ is reflected in the conjugacy problem of the group $G=\omega(H)$. So, in particular, if $H$ has an unsolvable word problem, $G$ has an unsolvable conjugacy problem. This observation plays a key part in the proof of Theorem A.

In fact the proof of Theorem A is carried out in several stages. We start out with a finitely presented group with an unsolvable word problem. We then modify it in order to obtain another finitely presented group $H$ with an unsolvable word problem all of whose defining relators are commutators. Using this group $H$ we adapt the Adian-Rabin construction, as in [13], to produce a family $H_{w}$ of finitely presented groups parametrized by the words $w$ in the given generators of $H$. Each of the $H_{w}$ is generated by the same number of generators and the given generators of $H_{w}$ freely generate $H_{w}$ modulo its derived group. The key fact here is that $H_{w}$ turns out to be free abelian if and only if $w={ }_{H} 1$ and hence has a solvable word problem. In the event that $w \neq \neq_{H} 1$, then $H_{w}$ has an unsolvable word problem. We now transform this family of groups $H_{w}$ by the word-conjugacy transformation $\omega$. Then by Corollary 5 on p. 28 of [12], the conjugacy problem for the group $G_{w}=\omega\left(H_{w}\right)$ is unsolvable whenever $w \neq \neq_{H}$ 1. The proof of Theorem A is completed by proving that if $w={ }_{H} 1$, then the groups $G_{w}$ are all isomorphic and have a solvable conjugacy problem; consequently the class of groups $G_{w}$ has an unsolvable isomorphism problem. The proof that the $G_{w}$ have a solvable conjugacy problem when $w={ }_{H} 1$ is the most difficult technical part of the proof and uses the same technology as that employed in [12].

1.5. The word-conjugacy transformation $\omega$. The word-conjugacy transformation $\omega$ was introduced in the second author's thesis [12]. The very nature of $\omega$ suggests that it might well have some other uses not only in combinatorial group theory but also in the study of associative and lie algebras. As already indicated it plays a key role in the proof of our main theorem.

We recall the details from [12], not only for later use but also to emphasise its importance. To this end, let

$$
P=\left\langle s_{1}, \ldots, s_{n} \mid r_{1}\left(s_{1}, \ldots, s_{n}\right), \ldots, r_{m}\left(s_{1}, \ldots, s_{n}\right)\right\rangle
$$

be a given finite presentation. Then we term the presentation $\omega(P)$ with generators

$$
s_{1}, \ldots, s_{n}, d_{1}, \ldots, d_{n}, t_{1}, \ldots, t_{m}, q
$$


and defining relations

$$
\begin{aligned}
d_{1}^{-1} q d_{1} & =s_{1} q s_{1}^{-1}, \ldots, d_{n}^{-1} q d_{n}=s_{n} q s_{n}^{-1}, \\
d_{i}^{-1} s_{j} d_{i} & =s_{j} \quad(1 \leq i, j \leq n), \\
t_{1}^{-1} q t_{1} & =q r_{1}\left(s_{1}, \ldots, s_{n}\right), \ldots, t_{m}^{-1} q t_{m}=q r_{m}\left(s_{1}, \ldots, s_{n}\right), \\
t_{i}^{-1} s_{j} t_{i} & =s_{j} \quad(1 \leq i \leq m, 1 \leq j \leq n)
\end{aligned}
$$

the word-conjugacy transform of (1). If $J$ is the group presented by $P$ and if $K$ is the group presented by $\omega(P)$, then we set $K=\omega(J)$ and we term it the word-conjugacy transform of $J$. It is these word-conjugacy transforms that are used in the proof of Theorem A.

\section{A modification of the Adian-Rabin construction}

2.1. Adjusting the presentation of a group with unsolvable word problem. We start with a finitely presented group

$$
A=\left\langle a_{1}, \ldots, a_{n} \mid r_{1}=1, \ldots r_{m}=1\right\rangle
$$

having unsolvable word problem. Of course each $r_{i}$ is a word on the $a_{j}$ and we sometimes write $r_{i}(\vec{a})$ or $r_{i}\left(a_{1}, \ldots, a_{n}\right)$ to make this explicit. Let $F_{a}$ be the free group on $a_{1}, \ldots, a_{n}$ and similarly let $F_{b}$ be the free group on $b_{1}, \ldots, b_{n}$. Now the obvious homomorphisms map $F_{a}$ and $F_{b}$ onto $A$. As observed by Mihaŭlova [11] (see also [13] or [8]), the fibre product of these two homomorphisms is the subgroup $S$ of $F_{a} \times F_{b}$ generated by the diagonal elements $a_{1} b_{1}, a_{2} b_{2}, \ldots, a_{n} b_{n}$ and the elements $r_{a}(\vec{a}), \ldots, r_{m}(\vec{a})$. (It follows that the $r_{i}(\vec{b}) \in S$ as well.) Thus $w(\vec{a}) \in S$ if and only if $w(\vec{a})={ }_{A} 1$. Since the word problem for $A$ is unsolvable, there is no algorithm to determine whether or not $w(\vec{a}) \in S$.

Next form the HNN extension $H$ of $F_{a} \times F_{b}$ by adding a stable letter $p$ which commutes with $S$. Then $p^{-1} w(\vec{a}) p=w(\vec{a})$ if and only if $w(\vec{a}) \in S$, so the word problem for $H$ is unsolvable. But we observe that $H$ can be presented as

$$
\begin{array}{r}
H=\left\langle a_{1}, \ldots, a_{n}, b_{1}, \ldots, b_{n}, p\right|\left[a_{i}, b_{j}\right]=1(1 \leq i, j \leq n), \\
\left.\left[p, a_{i} b_{i}\right]=1(1 \leq i \leq n),\left[p, r_{j}(\vec{a})\right]=1(1 \leq j \leq m)\right\rangle
\end{array}
$$

where we use the commutator notation $[x, y]=x^{-1} y^{-1} x y$. Thus we have established the following.

Lemma 1. There exists a finitely presented, torsion-free group $H$ with unsolvable word problem having a presentation in which all the defining relators are commutators. 
2.2. Modifying the Adian-Rabin construction. In what follows we need only the weaker result that the relators all lie in the derived group of the free group on the given generators. This can be established by other methods. For instance, if we embed the group $A$ with unsolvable word problem in a 2-generator group $B$ using the method of Higman, Neumann and Neumann [7], then the relators of $B$ all lie in the derived group of the free group on the generators given there.

The Adian-Rabin construction [13] yields a recursive family of finite presentations $J_{w}$ parametrized by words $w$ from a group $J$ with unsolvable word problem. The construction is arranged so that $J_{w}$ is the trivial group if and only if $w==_{J} 1$. Forming the free products $K_{w} * \mathbb{Z}^{n}$ with a free abelian group, we obtain a family of presentations which present a free abelian group of rank $n$ if and only if $w={ }_{J} 1$. But we need a stronger property, namely that when $w={ }_{J} 1$ the group should be free abelian with basis all of the generating symbols in the presentation. Here is a version of the construction which yields such a family.

Lemma 2 (Modified Adian-Rabin construction). Let $H$ be a group given by a finite presentation

$$
H=\left\langle x_{1}, \ldots, x_{n} \mid r_{1}=1, \ldots, r_{m}=1\right\rangle
$$

where the relators $r_{i}$ all lie in the derived group of the free group on the $x_{j}$. For any word $w$ in the given generators of $H$, let $H_{w}$ be the group with presentation obtained from the given one for $H$ by adding three new generators $a, b, c$ together with defining relations

$$
\begin{array}{rlrl}
a^{-1}[b, c] a & =[w, b], & & \\
a^{-1} c^{-2} b c^{2} a & =c^{-2} b c^{2}, & & \\
a^{-1} c^{-3} b^{-1} c b c^{3} a & =c^{-3} b^{-1} c b c^{3}, & & \\
a^{-1} c^{-2 \cdot 5^{i}}[b, c] c^{2 \cdot 5^{i}} a & =c^{-2 \cdot 5^{i}}\left[x_{i}, b\right] c^{2 \cdot 5^{i}}, & i & =1, \ldots, n, \\
a^{-1} c^{-3 \cdot 5^{i}}[b, c] c^{3 \cdot 5^{i}} a & =c^{-3 \cdot 5^{i}} b^{-1}\left[x_{i}, c\right] b c^{3 \cdot 5^{i}}, \quad i=1, \ldots, n, \\
a^{-1} c^{-5^{i}} x_{i} c^{5^{i}} a & =c^{-5^{i}} x_{i} c^{5^{i}}, & i & =1, \ldots, n \\
a^{-1} c^{-2^{i} \cdot 3^{j}} b c^{2^{i} \cdot 3^{j}} a & =c^{-2^{i} \cdot 3^{j}}\left[x_{i}, x_{j}\right] b c^{2^{i} \cdot 3^{j}}, & & 1 \leq i<j \leq n .
\end{array}
$$

Then

(i) if $w \neq_{H} 1, H$ is embedded in $H_{w}$ by the inclusion map on generators;

(ii) the abelianization $H_{w} /\left[H_{w}, H_{w}\right]$ is the free abelian group with basis the given generators $x_{1}, \ldots, x_{n}, a, b, c$;

(iii) the normal closure of $w$ in $H_{w}$ is the derived group $\left[H_{w}, H_{w}\right]$; in particular, if $w={ }_{H} 1$ then $H_{w}$ is free abelian on the given generators; 
(iv) if $H$ has an unsolvable word problem, then the isomorphism problem for the family of groups $H_{w}$ is unsolvable.

Proof. In view of the hypothesis on the defining relators of $H$, its abelianization $H /[H, H]$ is free abelian with basis the images of the $x_{i}(i=1, \ldots, n)$. In particular the $x_{i}$ have infinite order. Assertion (ii) is routine to check from the given relations since both sides of each equation have the same exponent sum on each generator.

Suppose now that $w \neq \neq_{H} 1$. Then in the ordinary free product $L=H *\langle c, b \mid\rangle$ of $H$ with the free group on generators $b$ and $c$, the element $[w, b]$ has infinite order. It is then easy to check that the right hand sides of the equations in (1) through (7) freely generate a free subgroup $D$ of $L$. Similarly the central parts of the left hand sides of (1) through (7) being conjugated by $a$ (that is, $[b, c], c^{-2} b c^{2}, \ldots$ ) freely generate a free subgroup $C$ of $L$. Thus if $w \neq_{H} 1$ we see that $H_{w}$ has the structure of an HNN extension of $L$ with stable letter $a$ which conjugates $C$ to $D$ as indicated (see Subsection 3.2). So $H$ is embedded in $H_{w}$. This proves (i).

To verify assertion (iii) we suppose $w={ }_{H} 1$. Then $[w, b]=1 \mathrm{in} L$ and by relation (1) it follows that $[b, c]=1$, so $b$ and $c$ commute. Consequently, relation (2) implies $a^{-1} b a=b$ and relation (3) implies $a^{-1} c a=c$ so that $a, b, c$ all commute with each other. The relations of type (4) and (5) then imply that $\left[x_{i}, b\right]=1$ and $\left[x_{i}, c\right]=1$ for $i=1, \ldots, n$. Hence by (6) it follows that $a^{-1} x_{i} a=x_{i}$ for $i=1, \ldots, n$. Finally the relations (7) imply that $\left[x_{i}, x_{j}\right]=1$ for $1 \leq i, j \leq n$. So all the generators of $H_{w}$ commute with each other and $H_{w}$ is free abelian on the given generators.

Finally to prove (iv), we observe that $H_{w}$ is free abelian of rank $n+3$ if and only if $w={ }_{H}$ 1. Since $H$ has an unsolvable word problem, we have proved (iv).

This completes the proof.

It follows immediately from Lemma 2 , once we choose $H$ to be a finitely presented group with unsolvable word problem in which all the relators lie in the derived group of the ambient free group, that we have proved the following theorem.

Theorem B. If $n$ is chosen sufficiently large, then there is no algorithm to determine of a finite presentation with $n$ generating symbols whether or not the group presented is free abelian of rank $n$.

2.3. A second look at Lemma 2. Lemma 2 will play a crucial role in the sequel. In order to formulate what we will require of Lemma 2 we adopt here the hypothesis and notation employed in its formulation. In addition we will assume that the word problem for $H$ is recursively unsolvable. As already noted, if $w={ }_{H} 1$, then $H_{w}$ is free abelian on the given generators and so has a solvable word problem. Hence there is no algorithm to determine for an arbitrary word $w$ whether or not $H_{w} \cong H_{1}$. Finally we note that $w=1$ (in the free group freely generated by given generators of $H$ ) if and only if $[w, b]=1$ in $H_{w}$. Moreover, if $w=1$, then $[w, b]=1$ is a 
consequence of the given relations $r_{1}=1, \ldots, r_{m}=1$ without using relations (1) through (7) in Lemma 2. Now observe that the parameter $w$ appears in exactly one relation of $H_{w}$ which can be rewritten as $[w, b] a^{-1}[c, b] a=1$ or as $u a^{-1}[c, b] a=1$ where $u=[w, b]$. Completely revising our notation we can summarize our results as follows:

Lemma 3. There is a recursive collection offinite presentations of groups $\mathrm{H}_{1}, \mathrm{H}_{2}, \ldots$, parametrised by the words $u_{1}, u_{2}, \ldots, u_{i}, \ldots$, of the form

$$
H_{i}=\left\langle s_{1}, \ldots, s_{n} \mid R_{1}\left(u_{i}\right)=1, R_{2}=1, \ldots, R_{m}=1\right\rangle
$$

with the following properties.

(1) Each $R_{i}$ lies in the derived group $\left[F_{S}, F_{S}\right]$ of the free group $F_{S}$ on the generators $s_{1}, \ldots, s_{n}$. Hence the groups $H_{i} /\left[H_{i}, H_{i}\right]$ are all free abelian on the listed generators.

(2) The first relation $R_{1}\left(u_{i}\right)=1$ depends on the parameter $u_{i}$ and has the form $u_{i} u_{0}=1$ where $u_{i}$ is a word depending on $i$ and $u_{0}$ is a fixed word.

(3) The relators $R_{2}, \ldots, R_{m}$ are fixed words, that is, they are the same for each $H_{i}$.

(4) $u_{1}$ is the empty word and $H_{1}$ is the free abelian group on the given generators.

(5) $H_{i}$ is isomorphic to $H_{1}$ if and only if $u_{i}=1$ in $H_{i}$.

(6) If $u_{i}=1$ in $H_{i}$, then this is a consequence of the relations $R_{2}=1, \ldots, R_{m}=1$.

(7) If $H_{i}$ is not isomorphic to $H_{1}$, then $u_{i} \neq 1$ in $H_{i}$ and $H_{i}$ has an unsolvable word problem.

(8) There is no algorithm to determine for an arbitrary $i \geq 1$ whether or not $H_{i}$ and $H_{1}$ are isomorphic.

We remark that the properties of the $H_{i}$ listed in the above statement are analogous to those of a corresponding class of groups for which triviality is undecidable that was used in [12] to prove that the isomorphism problem for finitely presented, residually finite groups is unsolvable.

Although not strictly necessary for what follows, it is useful to re-arrange the above presentations. We begin by transforming each of the relations (1) through (7) of Lemma 2. First we invert both sides of relations (2), (3) and (6). Then we insert 
inverse pairs and conjugate to bring them into the following forms.

$$
\begin{aligned}
a[b, w] a^{-1}[b, c] & =1, \\
{\left[b, c^{2}\right] a^{-1}\left[c^{2}, b\right] a[a, b] } & =1, \\
{\left[c, b c^{3}\right] a^{-1}\left[b c^{3}, c\right] a[a, c] } & =1, \\
c^{2 \cdot 5^{i}} a^{-1} c^{-2 \cdot 5^{i}}[c, b] c^{2 \cdot 5^{i}} a c^{-2 \cdot 5^{i}}\left[x_{i}, b\right] & =1, \quad i=1, \ldots, n, \\
b c^{3 \cdot 5^{i}} a^{-1} c^{-3 \cdot 5^{i}}[c, b] c^{3 \cdot 5^{i}} a c^{-3 \cdot 5^{i}} b^{-1}\left[x_{i}, c\right] & =1, \quad i=1, \ldots, n, \\
{\left[x_{i}, c^{5^{i}}\right] a^{-1}\left[c^{5^{i}}, x_{i}\right] a\left[a, x_{i}\right] } & =1, \quad i=1, \ldots, n, \\
b c^{2^{i} \cdot 3^{j}} a^{-1} c^{-2^{i} \cdot 3^{j}} b^{-1} c^{2^{i} \cdot 3^{j}} a c^{-2^{i} \cdot 3^{j}}\left[x_{i}, x_{j}\right] & =1, \quad 1 \leq i<j \leq n .
\end{aligned}
$$

Each of these relations $\left(1^{\prime}\right)$ through $\left(7^{\prime}\right)$ is equivalent to the corresponding relation in Lemma 2 in the sense that each has the same normal closure.

Observe that as the proof of Lemma 2, if $w={ }_{H} 1$ then relation $\left(1^{\prime}\right)$ implies $[b, c]=1$. Relations $\left(2^{\prime}\right)$ through $\left(5^{\prime}\right)$ have the forms $v_{2}[a, b]=1, v_{3}[a, c]=1$, $v_{4}\left[x_{i}, b\right]=1$ and $v_{5},\left[x_{i}, c\right]=1$ where each $v_{j}=1$ is a consequence of $[b, c]=1$. Thus $[a, b]=[a, c]=1$ and $\left[x_{i} b,\right]=\left[x_{i}, c\right]=1$. Then relations $\left(6^{\prime}\right)$ and $\left(7^{\prime}\right)$ have the form $v_{6}\left[a, x_{i}\right]=1$ and $v_{7}\left[x_{i}, x_{j}\right]=1$ where $v_{6}=1$ and $v_{7}=1$ are consequences of relations $[b, c]=1$ and $\left(2^{\prime}\right)$ through $\left(5^{\prime}\right)$. Finally the relations $r_{1}=1, \ldots, r_{m}=1$ are consequences of $[b, c]=1$ and $\left(2^{\prime}\right)$ through $\left(7^{\prime}\right)$.

Lemma 4. The relations $R_{1}, \ldots, R_{m}$ of the $H_{i}$ in Lemma 3 can be taken to have the following additional properties where $p=\frac{n^{2}-n}{2}$ :

(1) $u_{0}$ is $\left[s_{1}, s_{2}\right]$ and the relator $R_{1}$ of $H_{i}$ is $u_{i}\left[s_{1}, s_{2}\right]$;

(2) for each $j=2, \ldots, p$, the relation $R_{j}=1$ has the form $v_{j}\left[s_{k}, s_{\ell}\right]=1$ where the word $v_{j}$ is a consequence of $u_{0}=1$ and the earlier relations $R_{2}=1, \ldots, R_{j-1}=1$;

(3) for each $j=p+1, \ldots, m$, the relator $R_{j}$ is a consequence of $u_{0}=1$ and the earlier relations $R_{2}=1, \ldots, R_{p}=1$.

In addition the first relation of the group parametrized by the identity word $u_{1}$ is $R_{1}=1$, that is, $u_{0} u_{1}=u_{0}=\left[s_{1}, s_{2}\right]=1$.

\section{Construction of the residually nilpotent groups}

3.1. The groups $\boldsymbol{\omega}\left(\boldsymbol{H}_{\boldsymbol{i}}\right)$. We adopt now the notation and conclusions reached in the formulation of Lemma 4 . Thus we have a family of groups

$$
H_{i}=\left\langle s_{1}, \ldots, s_{n} \mid R_{1}\left(u_{i}\right)=1, R_{2}=1, \ldots, R_{m}=1\right\rangle \quad(i=1,2, \ldots)
$$


satisfying the conditions described in Lemma 4. We now apply the word-conjugacy transformation $\omega$ introduced in Section 1.5, to each of these groups. We need to describe the resultant groups in a little more detail. To this end, we observe that each $\omega\left(H_{i}\right)$ is a semi-direct product of the free group $F$ on the generators

$$
q, s_{1}, \ldots, s_{n}
$$

by the free group $T$ on the generators

$$
d_{1}, \ldots, d_{n}, t_{1}, \ldots, t_{m}
$$

The action of $T$ on $F$ is described as follows:

$$
\begin{aligned}
t_{i}^{-1} q t_{i} & =q R_{i}, & & 1 \leq i \leq m, \\
t_{i}^{-1} s_{j} t_{i} & =s_{j}, & & 1 \leq j \leq n, \\
d_{k}^{-1} q d_{k} & =s_{k} q s_{k}^{-1}, & & 1 \leq k \leq n, \\
d_{k}^{-1} s_{j} d_{k} & =s_{j}, & & 1 \leq j \leq n .
\end{aligned}
$$

Notice that only the relation $t_{1}^{-1} q t_{1}=q R_{1}\left(u_{i}\right)$ depends on the parameter $i$, or equivalently $u_{i}$, and it has the form $t_{1}^{-1} q t_{1}=q u_{i} u_{0}$ as noted in Lemma 4 .

We emphasize, as we observed earlier, the

Lemma 5. Each $G_{i}=\omega\left(H_{i}\right)$ is the split extension of the finitely generated free group $F$ by the finitely generated free group $T$. Hence each $G_{i}$ has solvable word problem and is residually finite.

Since each $R_{i}$ belongs to $\left[F_{S}, F_{S}\right]$, it follows that the action of each of the generators of $T$ on $F$ induces the trivial map on $F /[F, F]$. So, as noted already, on appealing to Lemma 2, p. 302 of [1] we find that

Lemma 6. $G_{i}$ is residually torsion-free nilpotent for $i=1,2, \ldots$

3.2. HNN extensions and the lemmas of Britton and Collins. We will need to look at the $G_{i}$ as HNN extensions (see [8] or [12] for discussions of HNN extensions and some relevant terminology). To this end observe that, because of the relations not involving $q$, the group $F_{S} \times T$ is a subgroup of $G_{i}$ and the presentation of $G_{i}$ can be obtained from one for $F_{S} \times T$ by adding the new generator $q$ and the defining relations

$$
\begin{aligned}
q^{-1} t_{i} q & =t_{i} R_{i}^{-1}, & & 1 \leq i \leq m, \\
q^{-1} d_{k} s_{k} q & =d_{k} s_{k}, & & 1 \leq k \leq n .
\end{aligned}
$$


Since the subgroups generated by the left and right sides of these equations are free on the listed generators, it follows that $G_{i}$ is also an HNN extension of $F_{S} \times T$ with stable letter $q$.

We can also view, somewhat trivially, the $G_{i}$ as HNN extensions with base group $F$, stable letters $t_{1}, \ldots, t_{m}, d_{1}, \ldots, d_{n}$ and associated subgroups all coinciding with the base group $F$ itself.

We remind the reader of some word and conjugacy terminology concerning HNN extensions (again, see [8] or [12]). Let $J=\langle S \mid D\rangle$ be a group and suppose that $\varphi: A \rightarrow B$ is an isomorphism between the subgroups $A$ and $B$ of $J$. Then the corresponding HNN extension $E$ with stable letter $p$ is presented as $E=\left\langle S, p \mid D, p^{-1} a p=\varphi(a), a \in A\right\rangle$. If $w$ is a word of $E$ of the form $w=w_{1} p^{-1} a p w_{2}$ with $a \in A$ then, using the defining relations, the subword $p^{-1} a p$ can be replaced by $b=\varphi(a)$ to obtain $w_{1} b w_{2}=G w$. Similarly a subword of the form $p b p^{-1}$ with $b \in B$ can be replaced by $a=\varphi^{-1}(b)$. In both cases the resulting word has 2 fewer occurrences of $p$ or $p^{-1}$. A subword of either of these forms is called a $p$-pinch and the process of replacing them using the defining relations as indicated is called pinching or $p$-reduction. A word is $p$-reduced if it does not contain a $p$-pinch. Britton's Lemma [3] says that if $w$ is a word of $E$ which contains $p$ or $p^{-1}$ and if $w={ }_{E} 1$ then $w$ contains a $p$-pinch. Recognizing $p$-pinches is equivalent to determining membership in the subgroups $A$ and $B$. So Britton's Lemma reduces the word problem for $E$ to the join of the word problem for $J$ and the membership problems for $A$ and $B$.

A word $w$ is cyclically $p$-reduced if every cyclic permutation of $w$ is $p$-reduced. Collins' Lemma [4] asserts that if $u, v \in E$ are cyclically $p$-reduced which are conjugate in $E$ and if $u$ begins with $p$, then there is a cyclic permutation $\bar{v}$ of $v$ which also begins with $p$ and an element $a \in A$ such that $a^{-1} u a={ }_{E} \bar{v}$. Moreover $u$ and $\bar{v}$ are $p$-parallel meaning they have the same sequence of $p^{ \pm 1}$ 's appearing in them. Under similar hypotheses, in case $u$ begins with $p^{-1}$ then for some $b \in B$ and cyclic permutation $\bar{v}$ of $v$ which also begins with $p^{-1}$ we have $b^{-1} u b={ }_{E} \bar{v}$. Similar versions of Britton's and Collins' lemmas apply when there are several stable letters.

We are now in a position to discuss the groups $G_{i}$ in more detail.

3.3. Relating conjugacy in $\boldsymbol{\omega}\left(\boldsymbol{H}_{\boldsymbol{i}}\right)$ to equality in $\boldsymbol{H}_{\boldsymbol{i}}$. The following result relates conjugacy in $G_{i}$ to equality in $H_{i}$ and partly explains the terminology that we have introduced here. Again the arguments given here are modeled on those in [12].

Lemma 7. If $w$ is any word on $\left\{s_{1}, \ldots, s_{n}\right\}$, then $q w$ is conjugate in $G_{i}$ to $q$ if and only if $w=H_{i}$ 1. Thus if $H_{i}$ has unsolvable word problem, then $G_{i}$ has unsolvable conjugacy problem. Moreover, $w=H_{i} 1$ if and only if $Y^{-1} q Y=G_{i} q w$ for some word $Y \in T$.

Proof. In one direction this claim is easy. For observe that the mapping which sends 
each $s_{i}$ in $G_{i}$ to the corresponding $s_{i}$ in $H_{i}$ and all other generators to the identity, defines a homomorphism $\phi$ from $G_{i}$ to $H_{i}$. Then if $Y^{-1} q Y=G_{i} q w$ it follows that $\phi(Y)^{-1} \phi(Y)={ }_{H_{i}} w$ and hence $w={ }_{H_{i}} 1$.

For the converse, suppose $w=H_{i} 1$. First observe that $t_{j}^{-1} q t_{j}=q R_{j}$ and hence $t_{j} q t_{j}^{-1}=q R_{j}^{-1}$. Also observe that $d_{k}^{-1} q s_{k} d_{k}=s_{k} q$. So if $x=x\left(s_{i}\right)$ is any word in the $s_{i}$ and $X\left(d_{i}\right)$ is the corresponding word in the $d_{i}$ then $X^{-1} q x X=x q$. Similarly, $X q X^{-1}=x^{-1} q x$ and $X^{-1} q X=x q x^{-1}$. Combining these observations we have

$$
\begin{aligned}
\left(X t_{j}^{\epsilon} X^{-1}\right)^{-1} q\left(X t_{j}^{\epsilon} X^{-1}\right) & =X t_{j}^{-\epsilon} X^{-1} q X t_{j}^{\epsilon} X^{-1}=X t_{j}^{-\epsilon} x q x^{-1} t_{j}^{\epsilon} X^{-1} \\
& =X x q R_{j}^{\epsilon} x^{-1} X^{-1}=q x R_{j}^{\epsilon} x^{-1}
\end{aligned}
$$

Since $w=H_{i} 1$,

$$
w={ }_{F_{S}} x_{1}\left(s_{i}\right) R_{j_{1}}^{\epsilon_{k}} x_{1}\left(s_{i}\right)^{-1} \ldots x_{k}\left(s_{i}\right) R_{j_{k}}^{\epsilon_{k}} x_{k}\left(s_{i}\right)^{-1}
$$

for suitable words $x_{k}$ in the given generators. So if we set

$$
Y=X_{k}\left(d_{i}\right) t_{j_{k}}^{\epsilon_{k}} X_{k}\left(s_{i}\right)^{-1} \ldots X_{1}\left(d_{i}\right) t_{j_{1}}^{\epsilon_{k}} X_{1}\left(s_{i}\right)^{-1}
$$

(note that the order has been reversed) it follows that $Y^{-1} q Y=G_{i} q w$ and of course $Y \in T$ as desired.

Corollary 8. If $u_{i}$ is the parameter in the first relation of $H_{i}$ and if $u_{i} \neq 1$ in $H_{i}$, then $G_{i}$ has an unsolvable conjugacy problem.

Actually the conjugacy problem for $G_{i}$ is at least as difficult as the conjugacy problem for $H_{i}$ as we see from the following lemma.

Lemma 9. If $u, v, x$ are any words on $\left\{s_{1}, \ldots, s_{n}\right\}$, then $q u$ is conjugate in $G_{i}$ to $q v$ if and only if $u$ and $v$ are conjugate in $H_{i}$. Moreover, $x^{-1} u x=H_{i} v$ if and only if $Y^{-1} x^{-1} q u x Y=G_{i} q v$ for some word $Y \in T$.

Proof. Since $H_{i}$ is a quotient of $G_{i}$ via $\phi, q u$ conjugate in $G_{i}$ to $q v$ clearly implies that $u$ and $v$ are conjugate in $H_{i}$. For the converse suppose that $x^{-1} u x=H_{i} v$. In $G_{i} q u$ is conjugate to $x^{-1} q u x$. Since $x$ is a word in the $\left\{s_{1}, \ldots, s_{n}\right\}$ we can conjugate $x^{-1} q u x$ by a word in the $d_{j}$ 's to $q x^{-1} u x$. Now set $w=v\left(x^{-1} u x\right)^{-1}$. Then $w=H_{i} 1$. It follows from Lemma 7 that there is a word $Y \in T$ with $Y^{-1} q Y=q w$. So we have $Y^{-1} q x^{-1} u x Y=q w x^{-1} u x=q v$. This proves the result.

A useful variant of Lemma 7 is the following:

Lemma 10. If $x, y$ are any words on $\left\{s_{1}, \ldots, s_{n}\right\}$, then $x y=G_{i} 1$ if and only if there is a word $Y \in T$ with $Y^{-1} q Y=x q y$. In this case $Y\left(t_{j}, d_{i}\right) x=Y\left(t_{j}, d_{i} s_{i}\right)$ and $Y\left(t_{j}, d_{i} s_{i}\right)^{-1} q Y\left(t_{j}, d_{i} s_{i}\right)=q y x$. 
Proof. If $Y^{-1} q Y=x q y$ with $Y \in T$, then applying the quotient map from $G_{i}$ to $H_{i}$ shows $x y=H_{i}$ 1. Conversely, if $x y={ }_{H_{i}} 1$ then by Lemma 7 for some $Y_{0} \in T$ we have $Y_{0}^{-1} q Y_{0}=q x y$. Now $d_{i}^{-1} q s_{i} d_{i}=s_{i} q$ so if $x=x\left(s_{i}\right)$ and $X=x\left(d_{i}\right)$ is the corresponding word in $d_{i}$ then $X^{-1} q x X=x q$. So letting $Y=Y_{0} X$ we have $Y^{-1} q Y=X^{-1} Y_{0}^{-1} q Y_{0} X=X^{-1} q x y X=x q y$. This equation can be rewritten as $Y^{-1} q^{-1} Y x q y=1$ and then by Britton's Lemma there is a $q$-pinch and so $Y x=Y\left(t_{j}, d_{i} s_{i}\right)$. This completes the proof.

We now proceed as in the proof of Theorem 26, pp. 74-77 of [12].

Lemma 11. If $u_{i}$ is the parameter in the first relation of $H_{i}$ and if $u_{i}=1$ in $H_{i}$, then the corresponding group $G_{i}$ is isomorphic to $G_{1}$.

Proof. Fix $i>1$ and suppose that $u_{i}=H_{i} 1$. Then $u_{i}=1$ is a consequence of the relations $R_{2}, \ldots, R_{m}$ by the properties of $H_{i}$. It follows from the proof of Lemma 7 that there is a word $Y$ on the generators $d_{1}, \ldots, d_{n}, t_{2}, \ldots, t_{m}$ such that $Y^{-1} q Y=q u_{i}$. Notice that $t_{1}$ is not included in the generators needed to express $Y$. Now $Y^{-1} q Y=q u_{i}$ holds in every one of the groups $G_{j}$ with $j>1$ since only the first relation $t_{1}^{-1} q t_{1}=q u_{j} u_{0}$ is different. Note that since $Y$ commutes with $F_{S}$, we have $Y q Y^{-1}=q u_{i}^{-1}$.

We now define a map $\varphi: G_{i} \rightarrow G_{1}$ which sends $t_{1}$ to $t_{1} Y$ and which fixes all of the other generating symbols. Note that $\varphi$ induces an automorphism of $T$ since $Y$ does not contain $t_{1}$. In $G_{1}$ we calculate

$$
\begin{aligned}
\varphi\left(t_{1}^{-1} q t_{1}\right) & =\left(t_{1} Y\right)^{-1} q\left(t_{1} Y\right)=Y^{-1} t_{1}^{-1} q t_{1} Y=Y^{-1} q u_{0} Y \\
& =Y^{-1} q Y u_{0}=q u_{i} u_{0}=\varphi\left(q u_{i} u_{0}\right) .
\end{aligned}
$$

Since $Y$ commutes with $F_{S}$, all the other relations are preserved and so $\varphi$ is a homomorphism.

Next we define a map $\left.\psi: G_{1}\right) \rightarrow G_{i}$ by $\psi\left(t_{1}\right)=t_{1} Y^{-1}$ and $\psi$ fixes all the other generating symbols. This time we compute in $G_{i}$ that

$$
\begin{aligned}
\psi\left(t_{1}^{-1} q t_{1}\right) & =\left(t_{1} Y^{-1}\right)^{-1} q\left(t_{1} Y^{-1}\right)=Y t_{1}^{-1} q t_{1} Y^{-1}=Y q u_{i} u_{0} Y^{-1} \\
& =Y q Y^{-1} u_{i} u_{0}=q u_{i}^{-1} u_{i} u_{0}=q u_{0}=\psi\left(q u_{0}\right) .
\end{aligned}
$$

Again all the other relations are preserved and so $\psi$ defines a homomorphism. Clearly $\varphi$ and $\psi$ are mutually inverse and so $G_{i}$ is isomorphic to $G_{1}$.

It would seem that we have nearly finished the proof of Theorem A. But it is still conceivable that $G_{1}$ is by "accident" isomorphic to one of the $G_{j}$ with $u_{j} \neq 1$ in $H_{j}$. If that were the case, then $G_{1}$ would also have unsolvable conjugacy problem by Corollary 8. So to complete the proof of Theorem A it suffices to show that $G_{1}$ has a solvable conjugacy problem. 
Of course conjugacy in $G_{1}$ is modelled on $H_{1}$ which is the free abelian group on the given generators. In fact, by using the technique of the previous lemma and some additional properties of the presentation for $H_{1}$ it is possible to prove the following

Lemma 12. $G_{1}$ is isomorphic to an analogous group $G_{0}$ in which all the $t_{i}^{-1} q t_{i}=q R_{i}$ relations have the very simple form $t_{i}^{-1} q t_{i}=q\left[s_{j}, s_{k}\right]$ or $t_{i}^{-1} q t_{i}=q$.

Since we will not use this presentation here, we omit the proof. However we note that the presentation of $G_{0}$ has only relations which say either that two symbols commute or, for the relations

$$
q^{-1} t_{i}^{-1} q t_{i}=\left[s_{\alpha}, s_{\beta}\right] \quad \text { and } \quad q^{-1} d_{k}^{-1} q d_{k}=q^{-1} s_{k}^{-1} q s_{k}
$$

that the commutator of two symbols is equal to the commutator of two other symbols. It would be astounding if such a group had an unsolvable conjugacy problem. One could hope to show there are no "accidental" isomorphisms with $G_{1}$ by some more direct technique. For instance, in each $G_{i}$ adding the relation $q=1$ maps $G_{i}$ onto $H_{i} \times T$. Now $H_{i} \times T$ has solvable word problem if and only $u_{i}=1$ in $H_{i}$. So if the normal closure of $q$ were characteristic, one could rule out an "accidental" isomorphism, but it is not clear whether this is true.

\section{Some elementary equations in free groups}

4.1. The equation $x^{k} u y^{k}=v$. In order to solve the conjugacy problem for the group $G_{1}$ we need a number of preliminary results. We recall first the theorem of Nielsen and Schreier [8], [10] that subgroups of free groups are free. Moreover Nielsen gave an algorithm for solving the generalized word problem in a free group. That is, if $F_{S}=\left\langle s_{1}, \ldots, s_{n} \mid\right\rangle$ is a free group, there is an algorithm to decide for words $w, h_{1}, \ldots, h_{k}$ on the $s_{i}$ whether or not $w$ belongs to the subgroup generated by $h_{1}, \ldots, h_{m}$. An easy graphical algorithm suitable for computer use is described in [15].

If $1 \neq u \in F_{S}$ and we write $u=z^{m}$ for $m \in \mathbb{Z}$ maximal, then it follows that $z$ generates the centralizer of $u$ in $F_{S}$. We call $z$ a primitive root of $u$ and note that $z^{-1}$ is the only other generator of the centralizer of $u$. Suppose that $1 \neq u, v \in F_{S}$ are two reduced words. If $u v=v u$, then $v$ belongs to the centralizer of $u$ and so $v=z^{k}$ for some $k$. In particular, $u$ and $v$ are powers of a common element and they have the same primitive root (up to inverses).

On the other hand if $u v \neq v u$ then $u$ and $v$ generate a non-abelian free subgroup $E$ and, since it has rank 2, $\{u, v\}$ is a free basis for $E$. Thus if $w \in F_{S}$ and $w \in H$ then $w$ is uniquely expressible as a word in $u$ and $v$. 
Lemma 13. There is an algorithm to determine of any four given words $x, y, u, v \in$ $F_{S}$ whether or not there exists an integer $k \in \mathbb{Z}$ such that

$$
x^{k} u y^{k}=v
$$

in $F_{S}$, and if so, to find $k$. If such a $k$ exists, it is unique except when $u=v$ and $y=u^{-1} x^{-1} u$ (and then the equation holds for all $k$ ).

Proof. Note that $x^{k} u y^{k}=v$ if and only if $x^{k} u y^{k} u^{-1}=v u^{-1}$, so if the equation has a solution for some $k$, then $v u^{-1}$ belongs to the subgroup $E_{0}$ generated by $x$ and $u y u^{-1}$. We now apply the algorithm to decide membership in $E_{0}$. If $v u^{-1}$ does not lie in $E_{0}, k$ does not exist. So we may assume that $v u^{-1}$ does lie in $E_{0}$ and that we have expressed $v u^{-1}$ as a word in the generators $x$ and $u y u^{-1}$.

In case $x$ and $u y u^{-1}$ do not commute, this representation of $v u^{-1}$ as a word in the generators is unique. By inspection we can see whether it has the correct form and if so determine $k$. In case $x=1$ or $y=1$ (but not both) then $v u^{-1}$ is a power of the non-trivial generator of $E_{0}$ and again $k$ is unique and can be determined by inspection.

Next suppose $x$ and $u y u^{-1}$ commute and neither is trivial. Let $z$ be a primitive root of $x$. Then for some $i, j$ we have $x=z^{i}$ and $u y u^{-1}=z^{j}$. To solve the desired equation we must have $z^{i k} z^{j k}=z^{k(i+j)}=v u^{-1}$. First suppose $v u^{-1} \neq 1$. Then once again, if such a $k$ exists, it is unique and has absolute value at most the length of $v u^{-1}$. This can easily be checked. Finally suppose $v u^{-1}=1$, that is, $u=v$. Then $j=-i$, and the original equation is $x^{k} u\left(u^{-1} x^{-1} u\right)^{k}=u$ which holds for every $k$. This completes the proof.

4.2. Solving a "simultaneous" conjugacy problem. Recall that an element $u \in F_{S}$ is said to be cyclically reduced if every cyclic permutation of $u$ is freely reduced. Two cyclically reduced words are conjugate if and only if one is a cyclic permutation of the other. Of course this solves the conjugacy problem for $F_{S}$. In more detail, if $u, v \in F_{S}$ are conjugate, then we can write $u=u_{0} u_{1} u_{0}^{-1}$ and $v=v_{0} v_{1} v_{0}^{-1}$ where the products are freely reduced as written and $u_{1}$ and $v_{1}$ are cyclically reduced. Also $u_{1}=u_{2} u_{3}$ and $v_{1}=u_{3} u_{2}$ where the products are freely reduced as written and we choose $u_{2}$ to be the shortest initial segment of $u_{1}$ with the indicated properties. We define the initial conjugator of $u$ to $v$ to be the word $x_{0}=u_{0} u_{2} v_{0}^{-1}$ and note the calculation

$$
x_{0}^{-1} u x_{0}=\left(v_{0} u_{2}^{-1} u_{0}^{-1}\right)\left(u_{0} u_{2} u_{3} u_{0}^{-1}\right)\left(u_{0} u_{2} v_{0}^{-1}\right)=v_{0} u_{3} u_{2} v_{0}^{-1}=v .
$$

Observe that the length of $x_{0}$ is at most the maximum of the lengths of $u$ and $v$. Now if $x$ is any solution of the equation $x^{-1} u x=v$ and we write $x=x_{0}\left(x_{0}^{-1} x\right)=x_{0} y$, then $x^{-1} u x=y^{-1} v y=v$. Thus $y$ commutes with $v$ and hence $y=z^{k}$ for some $k \in \mathbb{Z}$ where $z$ is a primitive root of $v$. We record these observations as follows: 
Lemma 14. If the two non-trivial elements $u, v \in F_{S}$ are conjugate, then every solution $x$ of the equation $x^{-1} u x=v$ has the form $x=x_{0} z^{k}$ where $x_{0}$ is the initial conjugator and $z$ is a primitive root of $v$ lem.

Using these observations we can now solve the "simultaneous" conjugacy prob-

Lemma 15. There is an algorithm to determine of any two sequences $u_{1}, \ldots, u_{m}$ and $v_{1}, \ldots, v_{m}$ of $m$ elements of $F_{S}$ whether or not there exists an element $x \in F_{S}$ such that the equations

$$
x^{-1} u_{1} x=v_{1}, x^{-1} u_{2} x=v_{2}, \ldots, x^{-1} u_{m} x=v_{m}
$$

hold simultaneously. If such an $x$ exists and $v_{1} \neq 1$, then either (1) $x$ is unique or (2) the initial conjugator $x_{0}$ for the equation $x^{-1} u_{1} x=v_{1}$ is a solution for the simultaneous system and the $v_{i}$ 's are all powers of the primitive root $z$ of $v_{1}$.

Proof. First check to see that each pair $u_{i}, v_{i}$ is conjugate. We can assume they are conjugate and are non-trivial. By Lemma 14 , every solution of the first equation $x^{-1} u_{1} x=v_{1}$ has the form $x=x_{0} z^{k}$ where $x_{0}$ is the initial conjugator of $u_{1}$ to $v_{1}$ and $z$ is a primitive root of $v_{1}$. So if we put $\bar{u}_{i}=x_{0}^{-1} u_{i} x_{0}$ for $i=2, \ldots, m$, then solving the original system is equivalent to finding a $k \in \mathbb{Z}$ such that

$$
z^{-k} \bar{u}_{2} z^{k}=v_{2}, \ldots, z^{-k} \bar{u}_{m} z^{k}=v_{m} .
$$

The elements $z, \bar{u}_{i}$ and $v_{i}$ are all fixed and so we can apply Lemma 13 to determine whether there is a $k$ such that the equation $z^{-k} \bar{u}_{2} z^{k}=v_{2}$ has a solution. We can assume that such a $k$ exists. Also by Lemma 13 this $k$ is unique except when $\bar{u}_{2}=v_{2}$ and $z=\bar{u}_{2}^{-1} z \bar{u}_{2}$, that is, except when $\bar{u}_{2}$ and $v_{2}$ are both powers of $z$ (and then the equation holds for all $k$ ). In the first instance we just check whether $z^{k}$ satisfies the remaining equations. Otherwise, we apply Lemma 13 successively to the remaining equations. Then at some stage we will either find a unique $k$ which does or does not yield a solution to the remaining equations or else we find that $\bar{u}_{i}=v_{i}$ and that the $v_{i}$ are all powers of $z$. In the event that the remaining $v_{i}$ are all powers of $z$, then $x$ conjugates the $u_{i}$ to the $v_{i}$ for all $i$. This completes the proof.

\section{Solution of the conjugacy problem for $G_{1}$}

5.1. Setting the stage for the proof. Our objective now is to find a solution of the conjugacy problem for the group $G_{1}$. This will complete the proof of our main result, Theorem A. For simplicity of notation we put $G=G_{1}$ and $H=H_{1}$. (We could use 
the even simpler presentation $G_{0}$ for $G_{1}$, but curiously that does not seem to be of much advantage and so we have not utilized it here.)

We recall that the group $H$ is modeled on the free abelian group on the $s_{i}$. The arguments given below work under the somewhat more general circumstances that $H$ is abelian. So the proof that $G$ has a solvable conjugacy problem will apply both in the case where $H$ is free abelian and in the case where it is trivial, which was the situation envisaged in the remark on p. 77 of [12]. So for the remainder of this section we will assume without further mention that $H$ is abelian. Consequently if $w \in\left[F_{S}, F_{S}\right]$ then $w={ }_{H} 1$; also $u$ and $v$ are conjugate in $H$ if and only if $u={ }_{H} v$. Now we can restate part of Lemma 9 as follows.

Lemma 16. Let $u$, $v$ be two words of $F_{S}$. Then qu is conjugate in $G$ to $q v$ if and only if $u={ }_{H} v$. Moreover, $u={ }_{H} v$ if and only if $Y^{-1} q u Y=q v$ for some word $Y \in T$.

5.2. Solving the conjugacy problem for $\boldsymbol{G}_{\mathbf{1}}$. We now apply these considerations to our group $G=G_{1}$ modeled on $H=H_{1}$ which we are viewing as an HNN extension in two different ways as discussed already in Subsection 3.2. We note that the relations involving the $t_{i}$ and $d_{j}$ acting on $F$ define $T$-pinches which preserve the number of $q$ symbols. On the other hand the relations for the stable letter $q$ acting on subgroups of $F_{S} \times T$ preserve the number of $t_{i}$ and $d_{j}$ symbols. We also note that there is an algorithm to recognize $q$-pinches since the associated subgroup is completely determined by the $T$ portion of the word. Hence for any word $w$ of $G$ we can effectively find a $(T, q)$-reduced word $\bar{w}$ with $w={ }_{G} \bar{w}$, that is, $\bar{w}$ is both $q$-reduced and reduced with respect to the stable letters that generate $T$. Similarly we can find a cyclically $(T, q)$-reduced word conjugate to $w$ in $G$.

We are now in a position to begin the proof of the last part of the proof of Theorem A.

Theorem 17. If the finitely presented group $H$ is abelian, then $G$ has solvable conjugacy problem.

Proof. Suppose we are given two words $U, V$ of $G$ and we want to determine whether or not they are conjugate. By the above discussion we can assume they are both cyclically $(T, q)$-reduced. There are several cases to consider, which we will deal with in turn.

5.3. Case (1): $\boldsymbol{U}$ is $(T, q)$-free. So here we are assuming that $U$ does not contain any $q$ or $T$ symbols, so it is an element of $F_{S}$. Then $V$ is also $(T, q)$-free by Collins' Lemma. Now any element $W$ of $G$ can be written as $A Y$ where $A \in F$ and $Y \in T$. But $W U W^{-1}=A Y U Y^{-1} A^{-1}=A U A^{-1}$ since the $T$ symbols commute with the $s_{i}$ 's. So if $U$ and $V$ are conjugate in $G$, they are already conjugate in $F$ and hence in $F_{S}$. Hence we can decide whether $U$ and $V$ are conjugate in this case. 
5.4. Case (2): $\boldsymbol{U}$ is $\boldsymbol{q}$-free and involves at least one $\boldsymbol{T}$ symbol. So here $U$ does not involve $q$ but does involves some $t_{i}$ or $d_{j}$. Then $V$ is also $q$-free and involves a $T$ symbol. After cyclically permuting $V$ and taking inverses of both if necessary, we can assume $U$ is $Y A$ and $V$ is $Y B$ where $Y=Y\left(t_{i}, d_{k}\right) \in T$ and $A, B \in F_{S}$. It suffices to test for this arrangement whether there is an element $W \in F$ with $W^{-1} U W=V$. We can easily check whether $U$ and $V$ are conjugate in $F_{S} \times T$, and so can assume they are not. Hence $W$ must involve a $q$ symbol. We can suppose $W$ is freely reduced and of the form $C q^{\epsilon} W_{1}$ where $C \in F_{S}$ and $\epsilon= \pm 1$. Now if

$$
W^{-1} U W=W_{1}^{-1} q^{-\epsilon} C^{-1} Y A C q^{\epsilon} W_{1}=V
$$

then $q^{-\epsilon} C^{-1} Y A C q^{\epsilon}$ is a $q$-pinch by Britton's Lemma. Hence, if $\epsilon=+1$, then we must have $C^{-1} Y A C=Y\left(t_{i}, d_{k} s_{k}\right)$ and $q^{-1} C^{-1} Y A C q=Y\left(t_{i} R_{i}^{-1}, d_{k} s_{k}\right)$ because of the form of the relations for $q$ as a stable letter. Similarly if $\epsilon=-1$, then we must have $C^{-1} Y A C=Y\left(t_{i} R_{i}^{-1}, d_{k} s_{k}\right)$ and $q C^{-1} Y A C q^{-1}=Y\left(t_{i}, d_{k} s_{k}\right)$. But similar considerations apply to $V=Y B$. So in order for $U=Y A$ and $V=Y B$ to be conjugate in $G$, one of them must be conjugate in $F_{S} \times T$ to $Y\left(t_{i}, d_{k} s_{k}\right)$ and the other to $Y\left(t_{i} R_{i}^{-1}, d_{k} s_{k}\right)$, and then the conjugation can be done by $q$ or $q^{-1}$. Hence we can decide whether $U$ and $V$ are conjugate.

5.5. Case (3): $\boldsymbol{U}$ is $\boldsymbol{T}$-free but involves $\boldsymbol{q}$. Then the same is true of $V$, and by taking inverses and cyclic permutations as necessary we may suppose they have the forms

$$
\begin{aligned}
& U=q u_{1} q^{\epsilon_{2}} u_{2} \ldots q^{\epsilon_{j}} u_{j}, \\
& V=q v_{1} q^{\epsilon_{2}} v_{2} \ldots q^{\epsilon_{j}} v_{j}
\end{aligned}
$$

where $u_{i}, v_{i} \in F_{S}$. Moreover we can arrange that, if circularly $U$ contains two consecutive $q$ symbols with the same sign, then $\epsilon_{2}=+1$. Otherwise either there is only one $q$ or the signs alternate.

By Collins' Lemma it suffices to determine whether these words are conjugate by a word of the form $W\left(t_{i}, d_{k} s_{k}\right)$. Now for any such word we can write $W\left(t_{i}, d_{k} s_{k}\right)=$ $W\left(t_{i}, d_{k}\right) x$ where $x=W\left(1, s_{k}\right) \in F_{S}$ and $W\left(t_{i}, d_{k}\right)^{-1} q W\left(t_{i}, d_{k}\right)=x q y$. Note that $x y={ }_{H} 1$.

Subcase (3.1): $j=1$ and so $U=q u_{1}$. Then $V$ is just $q v_{1}$ and, by Lemma $16, U$ and $V$ are conjugate if and only if $u_{1}={ }_{H} v_{1}$ which is decidable since $H$ is abelian.

Subcase (3.2): There is more than one $q$ symbol and $\epsilon_{2}=+1$. If hypothetically $W\left(t_{i}, d_{k} s_{k}\right)$ conjugates $U$ to $V$ then

$$
V=W\left(t_{i}, d_{k} s_{k}\right)^{-1} U W\left(t_{i}, d_{k} s_{k}\right)=q y u_{1} x q y u_{2} \ldots(x q y)^{\epsilon_{j}} u_{j} x
$$

and hence $v_{1}={ }_{F_{S}} y u_{1} x$. Then $u_{1}={ }_{H} v_{1}$ since $x y={ }_{H} 1$. Hence we can assume that $u_{1}={ }_{H} v_{1}$ and hence by Lemma 16 find a word $Y \in T$ such that $Y^{-1} q u_{1} Y=q v_{1}$. Now we conjugate $U$ by $Y$ to obtain

$$
\bar{U}=Y^{-1} U Y=q v_{1} q \bar{u}_{2} q^{\epsilon_{3}} \ldots q^{\epsilon_{j}} \bar{u}_{j}
$$


where $\bar{u}_{i} \in F_{S}$ are determined by the $u_{i}$ and the action of $Y$ on $q$. So we are reduced to determining whether $\bar{U}$ is conjugate to $V$ by a $W$ of the desired form.

If now hypothetically $W\left(t_{i}, d_{k} s_{k}\right)$ conjugates $\bar{U}$ to $V$ then

$$
V=W\left(t_{i}, d_{k} s_{k}\right)^{-1} \bar{U} W\left(t_{i}, d_{k} s_{k}\right)=q y v_{1} x q y \bar{u}_{2}(x q y)^{\epsilon_{3}} \ldots(x q y)^{\epsilon_{j}} \bar{u}_{j} x
$$

and hence $v_{1}=F_{S} y v_{1} x$ or equivalently $y=v_{1} x^{-1} v_{1}^{-1}$. So this equation becomes

$$
V=q v_{1} q v_{1} x^{-1} v_{1}^{-1} \bar{u}_{2}\left(x q v_{1} x^{-1} v_{1}^{-1}\right)^{\epsilon_{3}} \ldots\left(x q v_{1} x^{-1} v_{1}^{-1}\right)^{\epsilon_{j}} \bar{u}_{j} x .
$$

Comparing the right hand side with $V$ gives a series of equations depending on the signs $\epsilon_{i}$. For instance if $\epsilon_{3}=-1$ we would have $v_{2}=v_{1} x^{-1} v_{1}^{-1} \bar{u}_{2} v_{1} x v_{1}^{-1}$ while if $\epsilon_{3}=+1$ we would have $v_{2}=v_{1} x^{-1} v_{1}^{-1} \bar{u}_{2} x$. But for any sequence of signs $\epsilon_{i}$ these observations give a sequence of equations over $F_{S}$ of the form $\hat{v}_{i}=x^{-1} \hat{u}_{i} x$ for $i=2, \ldots, j$. By Lemma 15 we can determine whether a solution $x \in F_{S}$ to this system exists and if so find one. If no such solution exists, then $U$ and $V$ are not conjugate in $G$. On the other hand if $x$ is a solution, we also know $x v_{1} x^{-1} v_{1}^{-1}={ }_{H} 1$ since $H$ is abelian an so by Lemma 10 we can find a $W \in T$ such that $W^{-1} q W=x q v_{1} x^{-1} v_{1}^{-1}$ and hence $W\left(t_{i}, d_{k} s_{k}\right)$ conjugates $U$ to $V$. Thus we can decided conjugacy in this subcase.

Subcase (3.3): There are an even number of $q$ 's with alternating signs. If now hypothetically $W\left(t_{i}, d_{k} s_{k}\right)$ conjugates $\bar{U}$ to $V$ then

$$
V=W\left(t_{i}, d_{k} s_{k}\right)^{-1} U W\left(t_{i}, d_{k} s_{k}\right)=q y u_{1} y^{-1} q^{-1} x^{-1} u_{2} x q y \ldots q^{-1} x^{-1} u_{2 j} x .
$$

This gives two systems of conjugacy equations: for odd indices $y u_{i} y^{-1}=v_{i}$ and for even indices $x^{-1} u_{i} x=v_{i}$. Notice than none of the $u_{i}$ or $v_{i}$ can be equal to 1 since $U$ and $V$ are $q$-reduced.

Applying Lemma 15 we can decide whether solutions $x, y \in F_{S}$ to these systems exist. Hence we may assume we have found such solutions. If they are both unique, then $U$ and $V$ are conjugate if and only if $x y={ }_{H} 1$ which is decidable.

If one, say $y$, is unique but $x$ is not then the initial conjugator $x_{0}$ for the equation $x^{-1} u_{2} x=v_{2}$ solves the $x$-system and all the $v_{2 i}$ are powers of the primitive root $z_{2}$ of $v_{2}$. So $U$ and $V$ are conjugate if and only if $x_{0} z_{2}^{k} y={ }_{H} 1$ hold for some $k$, that is, if and only if $x_{0} y$ belongs to the subgroup of $H$ generated by $z_{2}$. Since $H$ is a finitely generated abelian group, there is an algorithm to determine whether such a $k$ exists. Similar considerations apply if $x$ is unique but $y$ is not unique.

We are left with the possibility that neither solution $x$ or $y$ is unique. In this case, the initial conjugators $x_{0}, y_{0}$ solve the respective systems and the $v_{2 i}$ are powers of the primitive root $z_{2}$ of $v_{2}$ and the $v_{2 i+1}$ are powers of the primitive root $z_{1}$ of $v_{1}$. Then $U$ and $V$ are conjugate if and only if $x_{0} z_{2}^{k} z_{1}^{\ell} y_{0}={ }_{H} 1$, that is, if and only if $x_{0} y_{0}$ belongs to the subgroup of $H$ generated by $z_{1}$ and $z_{2}$. Again this is decidable since $H$ is finitely generated and abelian. This complete this subcase. 
5.6. Case (4): $\boldsymbol{U}$ involves both $\boldsymbol{q}$ and $\boldsymbol{T}$ symbols. Then the same is true of $V$ and by taking inverses and cyclic permutations as necessary we may suppose they have the forms

$$
\begin{aligned}
& U=q u_{1} q^{\epsilon_{2}} u_{2} \ldots q^{\epsilon_{j}} u_{j} Y \\
& V=q v_{1} q^{\epsilon_{2}} v_{2} \ldots q^{\epsilon_{j}} v_{j} Y
\end{aligned}
$$

where $u_{i}, v_{i} \in F_{S}$ and $Y \in T$ is cyclically reduced. By Collins' Lemma it suffices to determine whether these words are conjugate by a word of the form $W\left(t_{i}, d_{k} s_{k}\right)$.

Now if we hypothetically suppose that $W\left(t_{i}, d_{k} s_{k}\right)^{-1} U W\left(t_{i}, d_{k} s_{k}\right)=V$ then we have $W\left(t_{i}, d_{k}\right)^{-1} Y W\left(t_{i}, d_{k}\right)=Y$ and so $W$ is a power of the primitive root $Z \in T$ of $Y$. Thus $U$ and $V$ are conjugate if and only

$$
Z\left(t_{i}, d_{k} s_{k}\right)^{-k} U\left(t_{i}, d_{k} s_{k}\right)^{k}=V
$$

for some $k \in \mathbb{Z}$. Here $Z \in T$ is fixed and $Z^{-1} q Z=x q y$ for fixed words $x, y \in F_{S}$. Also $Z^{-k} q Z^{k}=x^{k} q y^{k}$. So $U$ and $V$ are conjugate if and only if the equation

$$
q v_{1} q^{\epsilon_{2}} v_{2} \ldots q^{\epsilon_{j}} v_{j}=F_{S} q y^{k} u_{1}\left(x^{k} q y^{k}\right)^{\epsilon_{2}} u_{2} \ldots\left(x^{k} q y^{k}\right)^{\epsilon_{j}} u_{j} x^{k}
$$

has a solution $k \in \mathbb{Z}$ ( $x$ and $y$ are known). Now this gives a system of equations of the form $y^{k} u_{i} x^{k}=v_{i}$ or $y^{k} u_{i} y^{-k}=v_{i}$ or $x^{-k} u_{i} y^{-k}=v_{i}$ or $x^{-k} u_{i} x^{k}=v_{i}$ depending on the signs of the $e_{i}$. By Lemma 13, there is an algorithm to determine for each of these whether such a $k$ exists. For each equation the $k$ is unique or it holds for all $k$. Hence there is an algorithm to determine whether there is a $k$ for which all hold simultaneously, and then $U$ and $V$ will be conjugate. Either it is unique or the equations holds for all $k$, and then $U=V$. This completes the proof in this case and hence the proof of the theorem.

\section{References}

[1] G. Baumslag, Finitely generated residually torsion-free nilpotent groups. I. J. Austral. Math. Soc. Ser. A 67 (1999), 289-317. Zbl 0944.20021 MR 1716698

[2] G. Baumslag, Parafree groups. In Infinite groups: Geometric, combinatorial and dynamical aspects, Progr. Math. 248, Birkhäuser, Basel 2005, 1-14. MR 2195450

[3] J. L. Britton, The word problem. Ann. of Math. (2) 77 (1963), 16-32. Zbl 0112.25803 MR 0168633

[4] D. J. Collins, Recursively enumerable degrees and the conjugacy problem. Acta Math. 122 (1969), 115-160. Zbl 0175.27501 MR 0242671

[5] F. Grunewald and D. Segal, Some general algorithms. I: Arithmetic groups. Ann. of Math. (2) 112 (1980), 531-583. Zbl 0457.20047 MR 0595206 
[6] F. Grunewald and D. Segal, Some general algorithms. II: Nilpotent groups. Ann. of Math. (2) 112 (1980), 585-617. Zbl 0457.20048 MR 0595207

[7] G. Higman, B. H. Neumann, and Hanna Neumann, Embedding theorems for groups. J. London Math. Soc. 24 (1949) 247-254. Zbl 0034.30101 MR 0032641

[8] R. C. Lyndon and P. E. Schupp, Combinatorial group theory. Springer-Verlag, Berlin 1977. Zbl 0368.20023 MR 0577064

[9] W. Magnus, Beziehungen zwischen Gruppen und Idealen in einem speziellen Ring. Math. Ann. 111 (1935), 259-280. Zbl 0011.15201 MR 1512992

[10] W. Magnus, A. Karrass and D. Solitar, Combinatorial group theory. Wiley, New York 1966; reprint of the 1976 second edition, Dover, Mineola, NY, 2004. 0138.25604 MR 0207802

[11] K. A. Mihallova, The occurrence problem for the direct product of groups. Dokl. Akad. Nauk SSSR 119 (1958), 1103-1105. 0084.25302 MR 0100018

[12] C. F. Miller III, On group-theoretic decision problems and their classification. Ann. of Math. Stud. 68, Princeton University Press, Princeton, NJ, 1971. 0277.20054 MR 0310044

[13] C. F. Miller III, Decision problems for groups - survey and reflections. In Algorithms and classification in combinatorial group theory (ed. by G. Baumslag and C. F. Miller III), Math. Sci. Res. Inst. Publ. 23, Springer-Verlag, New York 1992, 1-59. 0752.20014 MR 1230627

[14] J. J. Rotman, An introduction to the theory of groups (4th edition). Grad. Texts in Math. 148, Springer-Verlag, New York 1995. 0810.20001 MR 1307623

[15] J. Stallings, Topology of finite graphs. Invent. Math. 71 (1983), 551-565. 0521.20013 MR 0695906

Received August 9, 2006

Department of Mathematics, City College of New York, Convent Ave. and 138th Street, New York, N.Y. 10031, U.S.A.

E-mail: gilbert@groups.sci.ccny.cuny.edu

Department of Mathematics and Statistics, University of Melbourne, Parkville 3052,

Australia

E-mail: c.miller@ms.unimelb.edu.au 